\title{
Endoscopic Ultrasound Guided Drainage of Peri-Rectal Collections: A
}

Salvage Approach

Yana Cavanagh, MD ${ }^{1}$, Kevin Hosein, $\mathrm{MD}^{2}$, Nihar Shah, MD, FACP ${ }^{3}$, Edward Milman, MD ${ }^{2}$, Sohail N. Shaikh, MD ${ }^{4}$

\section{Author Affiliations:}

1. Trinitas Regional Medical Center, Elizabeth, New Jersey

2. St. Joseph's Regional Medical Center, Paterson, New Jersey

3. Joan C Edwards School of Medicine, Marshall University, Huntington, West Virginia

4. New York Medical College, Valhalla, New York

All Authors have no conflict of interest to disclose.

\section{Corresponding Author:}

Yana Cavanagh, MD

Trinitas Regional Medical Center

Elizabeth, New Jersey

Email: ycavanagh@trinitas.org 


\section{Abstract}

Abdominal and pelvic abscesses can occur as a consequence of surgery and are associated with significant morbidity and mortality. It is well accepted that the primary treatment is antibiotic therapy and minimally invasive drainage. Surgical measures are typically reserved for patients with luminal perforations or those who do not respond to conservative measures. Previously available modalities of drainage involved percutaneous and computed tomography guided access. Recently, endoscopic ultrasound guided aspiration has emerged as a safe alternative for management of intra-abdominal abscesses.

Since its first application for peripancreatic drainage, endoscopic ultrasound techniques and technologies have significantly evolved. Endoscopic ultrasound interventions can now be performed quickly, safely and precisely, even outside of the operating room. Multiple reports and case series demonstrate the utility for endoscopic ultrasound guided drainage of fluid collections, providing they are in close proximity to the intestinal lumen. As a result, extension of this approach's indications has been considered.

Endoscopic ultrasound-guided drainage and stenting is an emerging approach which provides an accurate, efficient and safe alternative for the management of intra-abdominal collections. Furthermore, endoscopic ultrasound techniques may allow access to areas that are inaccessible or technically challenging with computed tomography guided or surgical approaches as illustrated in our case. Additionally, the internal nature of the access point may offer patients a more comfortable experience; and can facilitate preservation of enteral feeding routes or intestinal transit.

This case demonstrated the clinical complexity that often characterizes the management of postsurgical complications, particularly inflammatory collections. It further delineates the utility of Endoscopic ultrasound-fine needle aspiration techniques in the treatment of pelvic abscesses, even as a salvage therapy, where other approaches fail. EUS guided drainage should strongly be considered at multiple tiers of all treatment algorithms as a viable, and in some instances, preferable therapeutic modality for patients presenting with intra-abdominal collections.

Keywords: Endoscopic Ultrasound, Salvage therapy, EUS, Peri-Rectal, Collection, Abscess 


\section{Introduction}

Abdominal and pelvic abscesses can occur as a consequence of surgery and are associated with significant morbidity and mortality. (1) It is well accepted that the primary treatment is antibiotic therapy and minimally invasive drainage. Surgical measures are typically reserved for patients with luminal perforations or those who do not respond to conservative measures. (2) Previously available modalities of drainage involved percutaneous and computed tomography (CT) guided access. Recently, endoscopic ultrasound (EUS) guided aspiration has emerged as a safe alternative for management of intra-abdominal abscesses. $(3,4)$

One of the first applications of EUS was drainage of peripancreatic collections. (5) Since that time, EUS techniques have evolved and can now be performed quickly, safely and precisely, even outside of the operating room. (6) Successful drainage of gallbladder, perirectal, and splenic collections as well as bilomas have been reported. (2,7-9) Piraka et al. described 9 cases, in which EUS played a therapeutic role during the drainage of thoracic, abdominal, and pelvic collections. (10) Ramesh et al. compared transcolonic and transrectal approaches for EUS-guided abscess drainage. Similar to previous reports, they found no difference in procedural duration and attained successful drainage in all cases. (2)

These reports demonstrate the utility for EUS guided drainage of fluid collections, providing they are in close proximity to the intestinal lumen. (10) Additionally, EUS drainage allows for real-time access, under direct visualization. (2) As a result, extension of this approach's indications has been considered.

\section{Case Presentation}

We present the case of a 41-year-old Hispanic male that complained of a two-day history of severe pulsating suprapubic abdominal pain, 18 days after appendectomy. The pain was exacerbated by passing stool and partially alleviated by a combination of aluminum hydroxide and magnesium carbonate antacids. He also reported bloody, non-bilious vomiting, diarrhea, fever, chills as well as some dizziness. He had no history of recent travel or exposure to sick contacts. On physical examination, bowel sounds were normotympanic in all four quadrants. The abdomen was soft and non-distended but tender to palpation over the suprapubic region. There were no signs of rebound tenderness, ascites, hepatosplenomegaly or costovertebral angle tenderness.

An abdominal CT scan with contrast demonstrated a fluid collection with fat necrosis measuring 3.3 $\mathrm{cm}$ in the right lower quadrant, a pelvic abscess below the rectum measuring $5.6 \mathrm{~cm}$ (Figure 1), and a seminal vesicle abscess above the urinary bladder measuring $4 \mathrm{~cm}$. The patient underwent laparoscopy during which extensive adhesions involving the omentum, anterior abdominal wall, small bowel and sigmoid colon prevented access of the collections. The surgical procedure was aborted due to the dense adhesions. An interventional radiology (IR) guided drainage of the abscesses was also attempted, however, was also unsuccessful in draining the abscess cavity. Following failure of these approaches, salvage EUS guided drainage was pursued transrectally. 


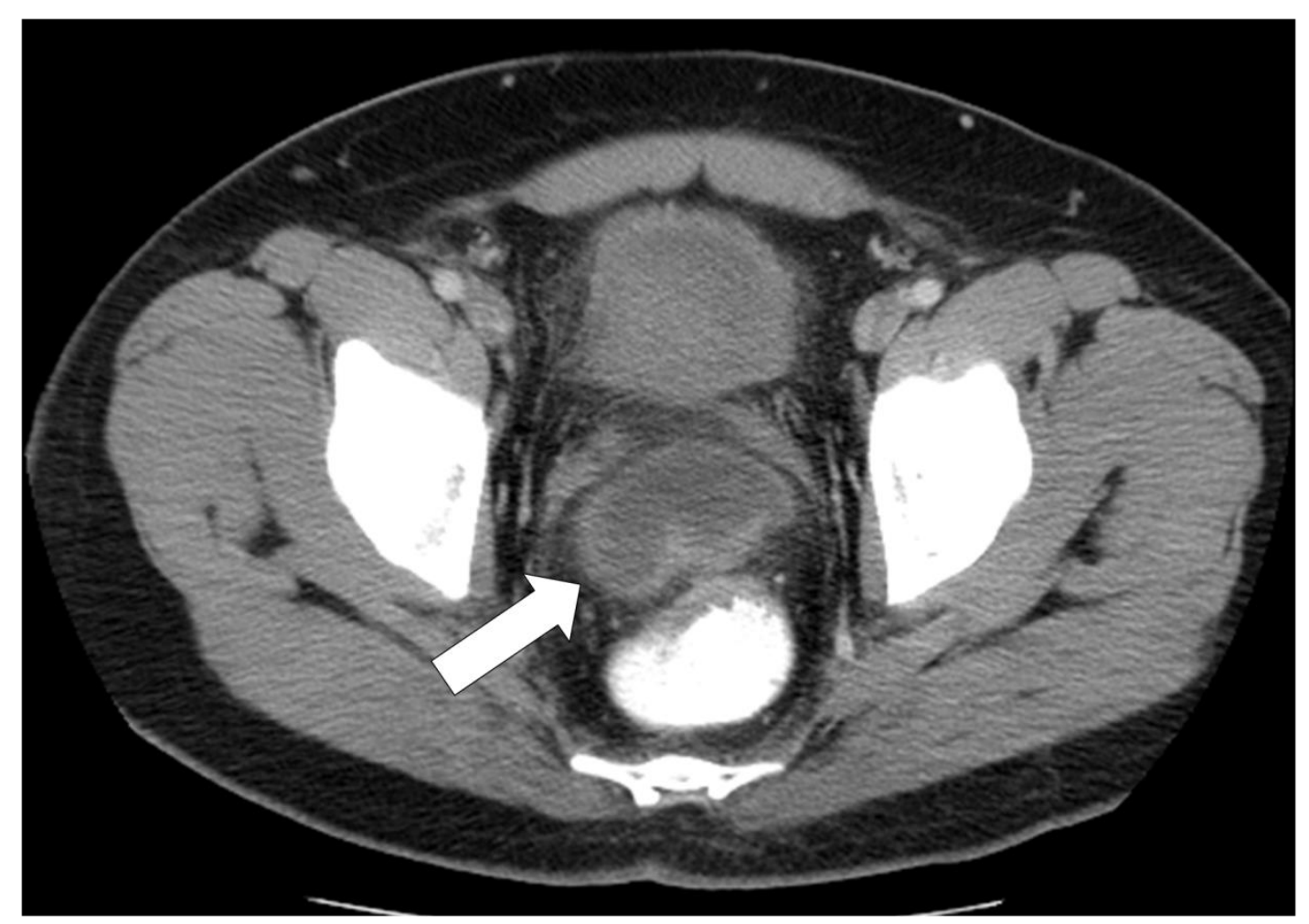

Figure 1: Axial CT of the pelvis: Oral contrast is noted in the rectum. A rim enhancing abscess (arrow) is visualized anterior to the rectum.

The abscess cavity was identified $<1 \mathrm{~cm}$ from the anterior wall of the colonic lumen and extended towards the right lower quadrant. Using a 19-gauge FNA needle the abscess cavity was punctured and $15 \mathrm{~mL}$ of purulent material was drained; following which, contrast was injected and the abscess margins were visualized with fluoroscopy. A guide wire was passed through the FNA needle and coiled in the abscess cavity under fluoroscopic guidance. An over-the-wire $10 \mathrm{~mm}$ x $4 \mathrm{~cm}$ dilation balloon (Boston Scientific - Hurricane RX) was used to dilate the tract and evacuate copious amounts of pus into the gastrointestinal lumen. Following complete decompression of the abscess cavity, a 10 French $\mathrm{x} 3 \mathrm{~cm}$ double pigtail stent was inserted to facilitate further drainage. The position of the stent was confirmed endoscopically and radiologically. The endoscope was withdrawn and the procedure was terminated without any complications.

Following EUS intervention our patient experienced resolution of his presenting symptoms.

Additionally, no new symptomatology was reported in association with the EUS intervention. A follow up CT scan demonstrated resolution of the abscess (Figure 2). The stent was subsequently removed by means of manual extraction, through the rectum. No additional endoscopic or surgical measures were required in the management of this case. 


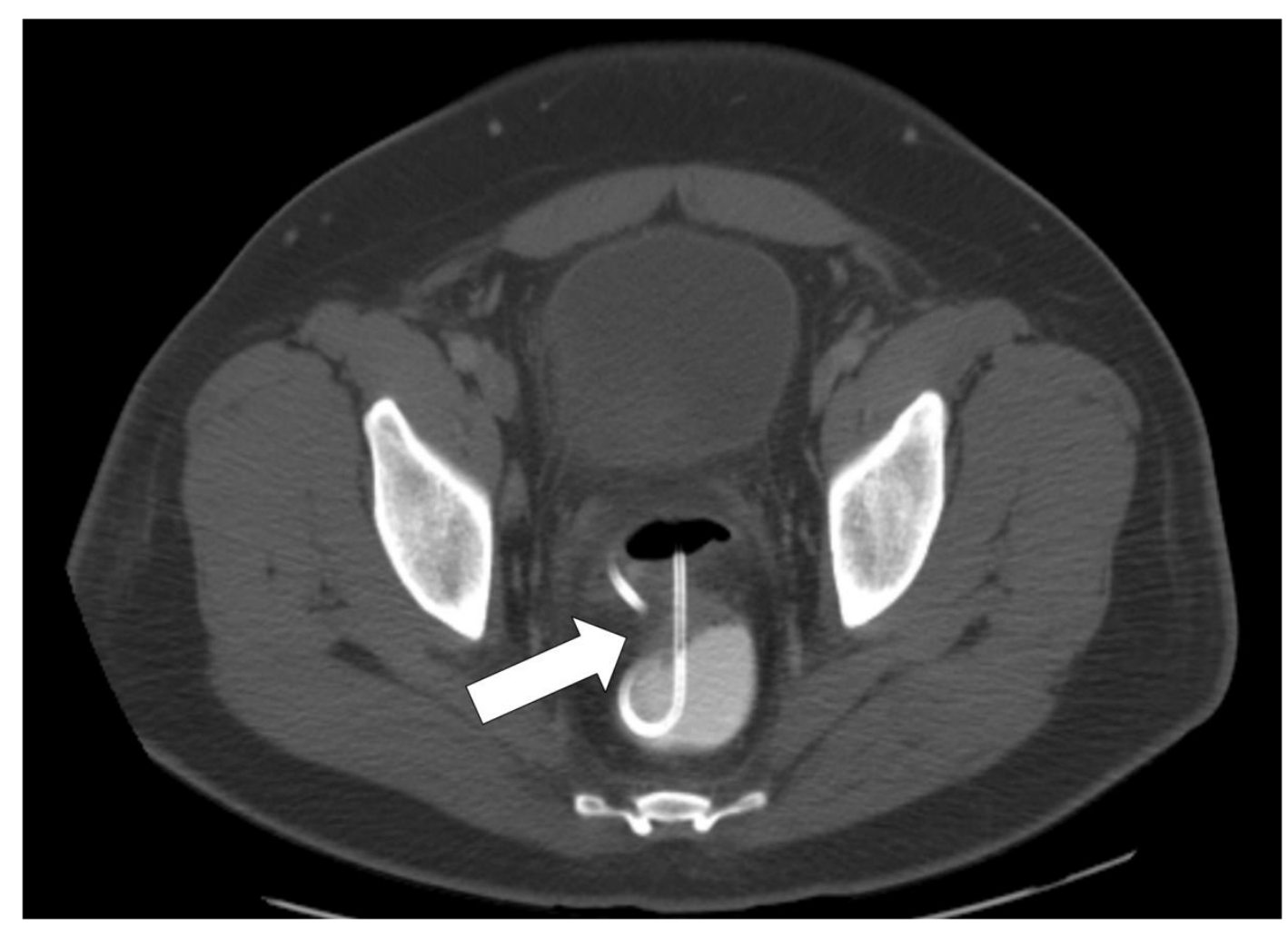

Figure 2. Axial CT of the pelvis in bone window: Transrectal catheter (arrow) is visualized within a drained abscess. Residual air is seen in the drained collection, next to the catheter.

\section{Conclusion}

EUS-guided drainage and stenting is an emerging approach which provides an accurate, efficient and safe alternative for the management of intra-abdominal collections. (11) Furthermore, EUS may allow access to areas that are inaccessible or technically challenging with CT guided or surgical approaches as illustrated in our case. Additionally, the internal nature of the access point may offer patients a more comfortable experience; and can facilitate preservation of enteral feeding routes or intestinal transit. $(11,12)$ Of note, EUS does require specialized equipment and may be technically challenging.

This case demonstrated the clinical complexity that often characterizes the management of post surgical complications, particularly inflammatory collections. It further delineates the utility of EUSFNA techniques in the treatment of pelvic abscesses, even as a salvage therapy in scenarios where surgical and CT guided approaches fail. EUS guided drainage should strongly be considered at multiple tiers of all treatment algorithms as a viable, and in some instances, preferable therapeutic modality for patients presenting with intra-abdominal collections. 


\section{References}

1. Ryan S, McGrath FP, Haslam PJ, Varghese JC, Lee MJ. Ultrasound-guided endocavitary drainage of pelvic abscesses: technique, results and complications. Clin. Radiol. 2003; 58: 75-9.

2. Ramesh J, Bang JY, Trevino J, Varadarajulu S. Comparison of outcomes between endoscopic ultrasound-guided transcolonic and transrectal drainage of abdominopelvic abscesses. J Gastroenterol Hepatol. 2013 Apr;28(4):620-5.

3. Varadarajulu S, Drelichman ER. Effectiveness of EUS in drainage of pelvic abscesses in 25 consecutive patients (with video). Gastrointest. Endosc. 2009; 70: 1121-7.

4. Giovannini M, Bories E, Moutardier V et al. Drainage of deep pelvic abscesses using therapeutic echo endoscopy. Endoscopy 2003; 35: 511-4.

5. Giovannini M, Bernardini D, Seitz JF. Cystogastrotomy entirely performed under endosonography guidance for pancreatic pseudocyst: results in six patients. Gastrointest Endosc 1998;48:200-203.

6. Vilmann P, Hancke S, Pless T, Schell-Hincke JD, Henriksen FW. One-step endosonography-guided drainage of a pancreatic pseudocyst: a new technique of stent delivery through the echo endoscope. Endoscopy 1998;30:730733.

7. Kahaleh M, Wang P, Shami VM, Tokar J, Yeaton P. Drainage of gallbladder fossa fluid collections with endoprosthesis placement under endoscopic ultrasound guidance: a preliminary report of two cases. Endoscopy 2005;37:393-396.

8. Lee DH, Cash BD, Womeldorph CM, Horwhat JD. Endoscopic therapy of a splenic abscess: definitive treatment via EUS-guided transgastric drainage. Gastrointest Endosc 2006;64:631-634.

9. Shami VM, Talreja JP, Mahajan A, Phillips MS, Yeaton P, Kahaleh M. EUS- guided drainage of bilomas: a new alternative? Gastrointest Endosc 2008;67: 136-140.

10. Piraka C, Shah RJ, Fukami N, Chathadi KV, Chen YK. EUS-guided transesophageal, transgastric and transcolonic drainage of intra-abdominal fluid collections and abscesses. Gastrointest Endosc. 2009 Oct;70(4):786-92.

11. Ulla-Rocha JL, Vilar-Cao Z, Sardina-Ferreiro R. EUS-guided drainage and stent placement for postoperative intraabdominal and pelvic fluid collections in oncological surgery. Therap Adv Gastroenterol. 2012 Mar;5(2):95-102.

12. Wehrmann T, Stergiou N, Voyel B, Riphaus A, Köckerling F, Frenz MB. Endoscopic debridement of paraesophageal mediastinal abscesses: a prospective case series. Gastrointest Endosc 2005;62:344-349. 